\title{
Editorial
}

\section{Changing the Face of Cardiovascular Trial Participation: Moving Beyond Middle-Aged White Guys}

\author{
David D. Waters, MD \\ Division of Cardiology, San Francisco General Hospital, and the Department of Medicine, University of California, San Francisco, California, USA
}

Clinical trials provide the most reliable data supporting the efficacy and safety of cardiovascular (CV) disease treatments. Generalizability is an important attribute of a clinical trial. In practical terms, you want to know that the patient you are about to treat was adequately represented in a trial demonstrating safety and efficacy. CV trials that exclude women or in which women are inadequately represented thus fall short of the ideal. This statement also holds true for older adults, people of color, and disadvantaged groups.

The tradition of excluding women from cardiovascular trials began long ago. A US Food and Drug Administration (FDA) guideline published in 1977 recommended that women of childbearing potential be excluded from phase 1 and early phase 2 clinical trials, and this directive is considered to have had a chilling effect on their access to phase 3 trials. ${ }^{1}$ In 1993, the FDA explicitly reversed their 1977 recommendation and called for trial data be analyzed to assess gender effect. ${ }^{1}$ The importance of $\mathrm{CV}$ trials for women was highlighted by the unexpected failures of hormone replacement therapy to reduce CV events in both primary and secondary prevention in postmenopausal women. ${ }^{2,3}$ Studies over the last 2 decades have broadened our understanding of sex and gender differences between women and men and how they might influence outcome differences for a variety of treatments.

\section{Participation Rates of Women in Cardiovascular Trials}

Four large studies were recently published documenting the rates of participation of women in $\mathrm{CV}$ trials. ${ }^{4-7} \mathrm{~A}$ study from the FDA's Office of Women's Health reported on trials supporting drug approvals from 2005 to 2015 . $^{4}$ The proportion of women enrolled overall was $46 \%$ (range, $22 \%$ $81 \%$ ). Participation to prevalence ratio (PPR), defined as the

Received for publication July 1, 2021. Accepted July 27, 2021.

Ethics Statement: Relevant ethical guidelines have been followed in the preparation of this review article.

Corresponding author: Dr David D. Waters, Division of Cardiology, Room 5G1, San Francisco General Hospital, 1001 Potrero Avenue, San Francisco, California 94110, USA. Tel.: +1-415-420-6646.

E-mail: David.Waters@ucsf.edu

See page $\mathrm{S} 3$ for disclosure information. percentage of women among trial participants divided by the percentage of women in the disease population, was within the desirable range of $0.8-1.2$ for atrial fibrillation (0.8-1.1), hypertension (0.9) and pulmonary hypertension (1.4), but was low for trials in heart failure (0.5-0.6), coronary disease (0.6), and acute coronary syndrome (0.6). Across these trials the authors found little indication of clinically meaningful gender differences in efficacy or safety.

In a larger, more recent report, the investigators studied 740 completed CV trials registered at ClinicalTrials.gov between 2010 and 2017. ${ }^{5}$ PPR was higher than 0.8 for hypertension and pulmonary hypertension, and lower (0.48 to 0.78) for arrhythmia, coronary disease, acute coronary syndrome, and heart failure trials. The most recent period, 20132017, saw increases in PPR for stroke $(P=0.007)$ and heart failure $(P=0.01)$ trials compared with the previous period.

The third large recent study included 60 randomized trials of lipid-lowering therapy with 485,409 participants reported from 1990 to 2018. ${ }^{6}$ Enrollment of women increased from $19.5 \%$ between 1990 and 1994 to $33.6 \%$ between 2015 and 2018. PPR for lipid trials of diabetes $(0.74)$, heart failure (0.27), stable coronary disease $(0.48)$, and acute coronary syndrome $(0.51)$ were low. However in trials of hypercholesterolemia, women were overrepresented (PPR 1.27).

In a study covering $598 \mathrm{CV}$ trials published between 1986 and 2015 in 3 major journals, the proportion of participants who were women increased from $21 \%$ in $1986-1990$ to $33 \%$ in 2011-2015.' As reported in the other 3 studies, women participated at lower rates than expected based on their proportion of the disease population.

The results of these 4 studies are roughly congruent, although details differ. Women participate less often than men, and their PPRs are lower for most conditions. Participation rates have improved more recently in studies where different periods were compared.

\section{Reasons for Low Rates of Trial Participation in Women}

A limited number of studies have investigated potential explanations for lower willingness to participate (WTP) in women. In a small Canadian study, $54 \%$ of 270 postmenopausal women stated that they would not participate in a $\mathrm{CV}$ trial, and $46 \%$ indicated that they would. ${ }^{8}$ Motivations 
reported for participating included personal health benefits $(82.2 \%)$, interest in research $(44.1 \%)$, and the possibility of benefiting society (29.1\%). Reasons for declining included personal illness (24.8\%), transportation issues (17.9\%), reluctance to increase medication (15.2\%), and concern about adverse effects (13.1\%).

In a study from northwest England in which patients with heart failure were approached by mail about study participation, male sex (odds ratio, 1.58; 95\% confidence interval [CI], 1.04-2.41) and younger age (odds ratio, 1.05; 95\% CI, 1.031.08) were associated with WTP.? The mean age of those accepting participation was 73.8 years compared with 78.9 years in those refusing. Main reasons given for refusal to participate were not feeling well enough $(36 \%)$, no transportation or inability to walk $(28 \%)$, not interested $(17 \%)$, old age $(10 \%)$, and too busy $(7 \%)$. These results may be specific to heart failure trials in which older age predominates.

In contrast, in an Italian study in which $59 \%$ of subjects expressed a WTP and 40\% did not, those who refused were more frequently women, were younger $(62 \pm 5$ vs $74 \pm 9$ years), and had a higher level of education and income. ${ }^{10}$ Among patients who refused, 629 responded to an interview. Reasons for refusal were the advice of family or friends $(28 \%)$, objection to trial procedures (placebo group, doubleblind allocation, 25\%), distrust of traditional medicine (20\%), uncertain about follow-up (10\%), did not want additional visits $(11 \%)$, and bad previous research experience (5\%). Different clinical conditions and different patient populations are likely to yield differences in reasons for nonparticipation.

In a randomized, double-blind study of 783 participants from 13 clinical centers, WTP was assessed for various trial scenarios. ${ }^{11}$ Key components of the trial scenarios, such as potential severity of adverse effects, trial sponsor, and amount of remuneration for participation were randomized. Women showed a lower distrust of medical researchers but perceived a greater risk of myocardial infarction and a greater risk from trial participation compared with men. Men were more willing to participate than women $(33.1 \%$ vs $28.7 \%$; relative risk, 1.15 ; $95 \%$ CI, 1.02-1.31). The sex difference in WTP disappeared with adjustment for perceived risks and benefits. Age, history of coronary disease, hypertension, and diabetes increased WTP in men but not in women. Monetary incentives had more effect on WTP in women than men $(P=$ 0.03 for sex interaction). The authors concluded that efforts to clarify perceptions of risks and benefits in men and women may help improve the sex disparity in WTP.

If women are less likely than men to enroll in CV trials, one might expect that they would also behave differently after they are enrolled, and one large study indicates that this is true. In a report from the TIMI (Thrombolysis in Myocardial Infarction) group including 135,879 men and 51,812 women (28\%) from 11 phase 3 or 4 trials, women had a higher rate of drug discontinuation compared with men (adjusted odds ratio, 1.22; 95\% CI, 1.16-1.28). ${ }^{12}$ Interestingly, this was true both in placebo and active treatment arms and was not due to baseline differences between the sexes. Adverse events accounted for drug discontinuation in 36\% of women and $36 \%$ of men. Women were more likely to withdraw consent compared with men (adjusted odds ratio, 1.26; 95\% CI, 1.17-1.36).

\section{Improving Participation Rates for Women}

Many CV trials aim to improve participation rates of women, and some set specific targets, yet in most cases these efforts are not serious and are not accompanied by any practical steps that are likely to make a difference. An initial practical step is to scrutinize inclusion and exclusion criteria to be certain that women are not being unintentionally excluded. Applying study entry criteria to a group of potential "real" study participants, men and women, can be a useful exercise to discover who will be excluded and why, before the study is initiated. Often initial entry criteria are too restrictive, recruitment lags, and entry criteria are then relaxed.

Women are underrepresented in leadership positions in $\mathrm{CV}$ trials. ${ }^{13}$ As a result, the orientation of trials tends to ignore the perspective of women. As previously noted, women have different issues related to trial participation compared with men, ${ }^{11}$ and it is reasonable to assume that these issues have not been adequately addressed when recruitment of women falls short. Although study coordinators are usually women, they may take their cues from male principal investigators and not be adequately trained to address the concerns of potential participants who are women.

Investigators who are serious about recruiting an adequate proportion of women into a trial should set a target and stop recruitment of men early if projections show that the target for women will not be met. In the recent heart failure trial in which this approach was adopted, $52 \%$ of participants were women $(\mathrm{n}=2,479)$ and a strong sex-by-treatment interaction was observed, with greater benefit in women than in men with heart failure and preserved ejection in the sacubitril plus valsartan group compared with the valsartan alone group (interaction $P<0.006) .{ }^{14}$

\section{Older Adults, People of Color, Disadvantaged Groups}

Most of the statements made about women in this review also apply to older adults and other subgroups of interest. Most older clinical trials had an upper age limit, with the result that scant or no trial data was available to guide decision-making in patients older than 75 or 80 years. Such patients comprise a rapidly growing proportion of the population. Because drug metabolism is slower and more variable in older adults, and for other reasons too, efficacy and safety might be different than for younger populations.

In the African-American Heart Failure Trial, self-identified Blacks with heart failure were randomly assigned to placebo or a fixed combination of hydralazine and isosorbide dinitrate. ${ }^{15}$ The trial was stopped early due to a $43 \%$ reduction in allcause mortality in the active treatment group.

The justification for limiting the trial to Blacks is that such patients respond less well to angiotensin-converting enzyme inhibitors than do non-Blacks.

Race is a poor marker of genetic differences among populations ${ }^{16}$; however, including different races in CV trials and reporting results by racial subgroup is important if the treatment under study will be used in that subgroup. Racial differences in outcomes may also be a result of environmental or behavioral differences between groups.

In Canada, CV risk is higher in certain racial and ethnic groups-South Asian, Afro-Caribbean, Chinese, and 
Hispanic, particularly in women. ${ }^{17}$ Canadians living in remote, rural, northern, or on-reserve locations; those with lower socio-economic status; and persons with disabilities also have an increased CV risk. Overcoming barriers and enrolling such patients in $\mathrm{CV}$ trials demonstrates inclusivity and is respectful of others.

It is tempting to conclude that the treatment of women, older adults, people of color, and other disadvantaged groups in clinical trials mirrors their treatment in the broader world. Adapting trials to the perspectives and needs of all potential subjects, not just middle-aged white guys, will make trial results more accurate and useful.

\section{Funding Sources}

The author has no funding sources to declare.

\section{Disclosures}

The author has no conflicts of interest to disclose.

\section{References}

1. U.S. Food and Drug Administration website. Gender studies in product development: historical overview. Available at: https://www.fda.gov/scienceresearch/womens-health-research/gender-studies-product-developmenthistorical-overview. Accessed June 21, 2021.

2. Grady D, Herrington D, Bittner V, et al. for the HERS Research Group. Cardiovascular outcomes during 6.8 years of hormone therapy. Heart and Estrogen/Progestin Replacement Study follow-up (HERS II). JAMA 2002;208:49-57.

3. Rossouw JE, Anderson GL, Prentice RL, et al. Risks and benefits of estrogen plus progestin in healthy postmenopausal women: principal results from the Women's Health Initiative randomized controlled trial. JAMA 2002;288:321-33.

4. Scott PE, Unger EF, Jenkins MR, et al. Participation of women in clinical trials supporting FDA approval of cardiovascular drugs. J Am Coll Cardiol 2018;71:1960-9.

5. Jin X, Chandramouli C, Allocco B, et al. Women's participation in cardiovascular clinical trials from 2010 to 2017. Circulation 2020;141: $540-8$.
6. Khan SU, Khan MZ, Raghu Subramanian C, et al. Participation of women and older participants in randomized clinical trials of lipidlowering therapies. JAMA Netw Open 2020;3:e205202.

7. Gong IY, Tan NS, Ali SH, et al. Temporal trends of women enrollment in major cardiovascular randomized clinical trials. Can J Cardiol 2019;35: 653-60.

8. Cheung AM, Lee Y, Kapral M, et al. Barriers and motivations for women to participate in cardiovascular trials. J Obstet Gynaecol Can 2008;30: $332-7$.

9. Lloyd-Williams F, Mair F, Shiels C, et al. Why are patients in clinical trials of heart failure not like those we see in everyday practice? J Clin Epidemiol 2003;56:1157-62.

10. Cosmi F, Mariottoni B, Tarquini B, et al. The patient in clinical research: disposable guinea pig or involved actor? G Ital Cardiol (Rome) 2020;21: 309-14.

11. Ding EL, Powe NR, Manson JE, Sherber NS, Braunstein JB. Sex differences in perceived risks, distrust, and willingness to participate in clinical trials: a randomized study of cardiovascular prevention trials. Arch Intern Med 2007;167:905-12.

12. Lau E, Braunwald E, Morrow DA, et al. Sex, permanent drug discontinuation, and study retention in clinical trials. Insights from the TIMI trials. Circulation 2021;143:685-95.

13. Denby KJ, Szpakowski N, Silver J, et al. Representation of women in cardiovascular clinical trial leadership. JAMA Intern Med 2020;180: $1382-3$.

14. Solomon SD, McMurray JJV, Anand IS, et al; PARAGON-HF Investigators and Committees. Angiotensin-neprilysin inhibition in heart failure with preserved ejection fraction. N Engl J Med 2019;381: 1609-20.

15. Taylor AL, Ziesche S, Yancy C, et al. Combination of isosorbide dinitrate and hydralazine in blacks with heart failure. N Engl J Med 2004;351: 2049-57.

16. Bloche MG. Race-based therapeutics. N Engl J Med 2004;351:2035-7.

17. Jaffer S, Foulds HJA, Parry M, et al. The Canadian Women's Heart Health Alliance ATLAS on the epidemiology, diagnosis, and management of cardiovascular disease in women - chapter 2: scope of the problem. CJC Open 2020;3:1-11. 